\title{
Indices of renal tubular function in perinatal asphyxia
}

\author{
Frank Willis, Julie Summers, Corrado Minutillo, Ian Hewitt
}

\begin{abstract}
Aims-To determine and compare two urinary indices of renal tubular function, $\boldsymbol{N}$-acetyl-glucosaminidase (NAG) and $\beta_{2}$ microglobulin $\left(\beta_{2} M\right)$, in healthy term neonates and babies with perinatal asphyxia.

Methods-In a prospective case-control study using asphyxiated $(n=35)$ and normal control $(n=55)$ infants, urinary NAG and $\beta_{2} M$ were assayed at 24-48 hours of life, 4-6 days, and 4-6 weeks.

Results-NAG and $\beta_{2} M$ were significantly increased at 24-48 hours and 4-6 days in the asphyxiated infants compared with the controls. Increased NAG values reflect the degree of perinatal asphyxia more than do $\beta_{2} M$. Gentamicin also increased NAG excretion, but to a lesser extent than did perinatal asphyxia.

Conclusions-NAG $\left(+/-\beta_{2} M\right)$ may be a useful marker of perinatal asphyxia. Urinary NAG concentrations correlate with the severity of perinatal asphyxia. (Arch Dis Child 1997;77:F57-F60)
\end{abstract}

Keywords: perinatal asphyxia; renal tubular function; $\mathrm{N}$-acetyl-glucosaminidase

Perinatal asphyxia causing organ damage is an important neonatal problem. Its study is challenging because it is difficult to measure. The kidney is very sensitive to ischaemic damage. ${ }^{1}$ Fetal and neonatal "asphyxia" are the main causes of transient renal impairment or acute renal failure in neonates. ${ }^{23}$

Urinary concentrations of $\mathrm{N}$-acetylglucosaminidase (NAG) and $\beta_{2}$-microglobulin $\left(\beta_{2} M\right)$ are sensitive indices of renal tubular function. NAG is a lysosomal enzyme present in proximal tubular cells. ${ }^{4}$ Its molecular weight precludes filtration at the glomerulus but it is rapidly cleared from the circulation by the liver. Increased urinary excretion is a consequence of renal tubular cell breakdown. ${ }^{5} \beta_{2} M$ is a low molecular weight protein, freely filtered through the glomerulus, but almost totally reabsorbed in the proximal tubules. ${ }^{6}$ Urinary $\beta_{2} M$ concentration increases with degree of tubular dysfunction.

The objective of this study was to compare concentrations of urinary NAG and $\beta_{2} M$ in a population of asphyxiated and normal term neonates over the first 4 to 6 weeks of life.

\section{Methods}

This prospective case-control study compared 35 term babies with perinatal asphyxia (group 1) with a group of 55 normal term infants (group 2). Informed consent was obtained from the parents of all participants. Single "spot" urine samples were collected using a standard paediatric urine bag at 24 to 48 hours, 4 to 6 days, and 4 to 6 weeks of life. Group 1 subjects had urine samples tested daily during the period of hospital admission for the presence of haematuria and routine serum biochemistry (including creatinine concentrations) performed at least daily for the first week.

Group 1 comprised 35 consecutive term babies with perinatal asphyxia transferred from maternity hospitals to a level 3 neonatal intensive care unit at a paediatric hospital (Princess Margaret Hospital for Children, Perth, Western Australia). Perinatal asphyxia was defined as a 5 minute Apgar score of 5 or less plus fulfilment of the diagnostic criteria of posthypoxic/ischaemic encephalopathy according to the clinical staging system of Sarnat ${ }^{7}$ (table 1). Each case was assigned a Sarnat score, reflecting the degree of severity. Prenatal signs of fetal distress, including meconium stained liquor and fetal heart rate abnormalities, were sought to confirm the clinical impression, as were umbilical cord blood gas values if available. Cases were retrospectively reassessed after discharge or post mortem examination and excluded if pathology other than perinatal asphyxia was suggested.

Group 2 comprised normal term infants $(n=55)$ recruited from a suburban maternity hospital (Woodside Maternity Hospital, East Fremantle, Western Australia). Subjects in this group had 5 minute Apgar scores of more than 6 , normal behaviour over the first week of life, and were classified as normal on routine clinical examination.

To identify the possible influence of amino glycoside antibiotics on results for group 1, urine samples at 24 to 48 hours of life were obtained and analysed for NAG from 13 babies consecutively admitted with transient tachypnoea of the newborn (TTN), but no evidence of perinatal asphyxia, and 12 further infants with perinatal asphyxia (as defined before). These subjects form groups $3 a$ and $3 b$, respectively. Infants in both groups were routinely treated with antibiotics (gentamicin and ampicillin) for at least the first 24 hours of life, at which time urine samples were collected. Gentamicin concentrations were measured and those with raised values excluded from analysis. Further data on the subjects are given in table 2.

Samples were frozen at -20 to $-70^{\circ} \mathrm{C}$, until assay, when the $\mathrm{pH}$ was adjusted to $6-8$. 
Table 1 Sarnat score: clinical staging of posthypoxic encephalopathy

\begin{tabular}{llll}
\hline Factor & Stage 1 & Stage 2 & Stage 3 \\
\hline Consciousness & Alert & Lethargic & Coma \\
Tone & Normal & Hypotonic & Flaccid \\
Tendon reflexes & Increased & Increased & Depressed \\
Myoclonus & Yes & Yes & No \\
Suck & Active & Weak & Absent \\
Moro response & Increased & Incomplete & Absent \\
Grasp reflex & Normal & Increased & Absent \\
Oculocephalic & Normal & Increased & Depressed \\
Pupils & Dilated & Constricted & Variable/fixed \\
Respiration & Regular & Periodic/variable & Apnoea \\
Heart rate & Increased/normal & Bradycardia & Bradycardia \\
Seizures & No & Common & Uncommon \\
EEG & Normal & Periodic or paroxysmal & Periodic or isoelectric \\
\hline
\end{tabular}

Table 2 Clinical data on neonates studied

\begin{tabular}{llll}
\hline Group & $\begin{array}{l}\text { Gestational age } \\
\text { (weeks) }\end{array}$ & Birthweight $(g)$ & 5 minute Apgar score \\
\hline $1(\mathrm{n}=35)$ Perinatal asphyxia & $36-42(39)$ & $\star 1720-4970(3307)$ & $1-4(3)$ \\
$2(\mathrm{n}=55)$ Healthy full term & $37-42(40)$ & $2480-4990(3584)$ & $9-10(9)$ \\
$\begin{array}{l}\text { 3a }(\mathrm{n}=13) \text { Transient } \\
\text { tachypnoea }\end{array}$ & $36-40(38)$ & $2200-3560(3110)$ & $7-9(9)$ \\
$\begin{array}{l}\text { 3b }(\mathrm{n}=12) \text { PA paired with } \\
\text { TTN }\end{array}$ & $36-42(39)$ & $2575-4045(3440)$ & $1-4(3)$ \\
\hline
\end{tabular}

All data are presented as mean and range; ${ }^{\star}$ Two infants were small for gestational age, one was a twin.

Urinary $\beta_{2} M$ was measured using a competitive enzyme immunoassay (T-Cell Diagnostics Inc., Cambridge, MA, USA). Specimens containing $\beta_{2} \mathrm{M}$ were incubated with rabbit antihuman $\beta_{2} M$ antibody and with horseradish peroxidase labelled human $\beta_{2} M$ to compete for a limited number of binding sites. During incubation, the antibody simultaneously bound to the microtitre plate, which had been coated with an antigen to anti- $\beta_{2} M$. Unbound conjugate was removed by washing, and the remaining enzyme-labelled antigen incubated with 3,3'-5,5'-tetramethylbenzidine substrate. The reaction was terminated by addition of 2 molar $\mathrm{H}_{2} \mathrm{SO}_{4}$, and the absorbency (inversely proportional to the concentration of $\beta_{2} M$ in the sample) was measured at $450 \mathrm{~nm}$. Results were expressed as milligrams per litre.

NAG was determined colorimetrically by measuring the release of 3-cresol purple from the substrate 3-cresolsulphonphthaleinyl- $N$ acetyl-B-D-glucosaminide (Boehringer Mannheim, Germany). Results were expressed as International Units per millimole of creatinine. Serum and urine creatinine concentrations were measured using a dry chemistry analyser, Kodak Ektachem 500 (Eastman Kodak, Rochester, New York). Haematuria was assessed using standard dipstick reagent strips (Multistix, Bayer Diagnostics, Victoria).

Statistical analysis was performed using INSTAT (Instant Biostatics, version 2). Mean, median, standard deviations, and 95\% confidence limits are reported and were tested using the Kruskal-Wallis non-parametric ANOVA test and the Mann-Whitney U test, giving a two tailed $P$ value. Non-parametric statistics were applied as logarithmic transformation failed satisfactorily to normalise the data.

The study was approved by the Research and Ethics Committee of Princess Margaret Hospital for Children.

\section{Results}

The postnatal progression in urinary NAG and $\beta_{2} \mathrm{M}$ concentrations are shown in table 3 . Initially raised concentrations of both NAG and $\beta_{2} \mathrm{M}$ in the perinatal asphyxia group returned to control group values by 4 to 6 weeks of life.

Table 4 shows the effect of gentamicin on urinary NAG at 24 to 48 hours of life in groups 2, 3a, and 3b. Median NAG concentrations for TTN (group 3a) and perinatal asphyxia (groups 1 and 3b) subjects differed significantly from those of normal controls (group 2) $(\mathrm{P}<0.0001)$. The perinatal asphyxia group had significantly higher values than did the TTN group $(P=0.02)$.

The correlation between median urinary NAG concentrations at 24 to 48 hours of life and the degree of perinatal asphyxia, as assessed by the Sarnat score, is shown in table 5. There was an increase in mean urinary NAG excretion with increasing severity of perinatal asphyxia. Median values for the control group differed significantly from those of Sarnat 1 $(P=0.0004)$, Sarnat $2(P<0.0001)$, and Sarnat $3(P=0.0026)$ groups. There was also a positive correlation which was not significant for NAG at 4 to 6 days; this was not seen with $\beta_{2} \mathrm{M}$ at 24 to 48 hours or 4 to 6 days (data not shown).

Dipstick testing for haematuria in the perinatal asphyxia group over the first 4 days of life revealed "large" blood on at least one occasion in $14 / 32$, "trace" or "small" in 5/32 and no blood in 13/32 infants. No information was recorded for three infants.

The mean serum creatinine concentration for the perinatal asphyxia group was 101.1

Table $3 \quad \beta_{2} M$ and $N A G$ values over time

\begin{tabular}{|c|c|c|c|c|c|c|}
\hline & \multicolumn{3}{|l|}{$\beta_{2} M$} & \multicolumn{3}{|l|}{$N A G$} \\
\hline & 24-48 hours & 4-6 days & 4-6 weeks & 24-48 hours & 4-6 days & 4-6 weeks \\
\hline \multicolumn{7}{|c|}{ Control group } \\
\hline Number & 35 & 34 & 25 & 55 & 52 & 41 \\
\hline Mean & 1.17 & 1.55 & 0.34 & 1.89 & 3.50 & 2.57 \\
\hline SD & 1.33 & 1.26 & 0.47 & 1.21 & 3.36 & 1.86 \\
\hline Median & 0.66 & 1.60 & 0.13 & 1.60 & 2.60 & 2.10 \\
\hline $95 \% \mathrm{CI}$ & $0.71-1.62$ & $1.12-1.99$ & $0.15-0.53$ & $1.56-2.22$ & $2.56-4.43$ & $1.89-3.16$ \\
\hline \multicolumn{7}{|l|}{$P A$ group } \\
\hline Number & 34 & 24 & 10 & 35 & 23 & 10 \\
\hline Mean & 7.85 & 10.60 & 0.34 & 28.84 & 29.31 & 2.20 \\
\hline SD & 11.57 & 14.33 & 0.51 & 65.56 & 85.57 & 1.27 \\
\hline Median & 3.88 & 3.69 & 0.13 & 9.60 & 8.30 & 1.85 \\
\hline $95 \% \mathrm{CI}$ & $3.81-11.90$ & $4.59-16.61$ & $-0.02-0.70$ & $3.30-48.38$ & $-7.70-66.31$ & $1.29-3.11$ \\
\hline$P$ value & $<0.0001$ & $<0.0005$ & 0.985 & $<0.0001$ & $<0.0001$ & 0.469 \\
\hline
\end{tabular}


Table 4 Gentamicin effects on 24-48 hour NAG concentrations

\begin{tabular}{llll}
\hline & Control (group 2) & $\begin{array}{l}\text { TTN (gentamicin) (group } \\
\text { 3a) }\end{array}$ & $\begin{array}{l}\text { PA (gentamicin) (groups 1 and } \\
3 b)\end{array}$ \\
\hline Number & 55 & 13 & $47^{\star}$ \\
Mean & 1.89 & 5.03 & 22.40 \\
SD & 1.21 & 2.78 & 56.93 \\
Median & 1.60 & 4.00 & 9.60 \\
$95 \%$ CI & $1.56-2.22$ & $3.35-6.71$ & $5.68-39.14$ \\
\hline
\end{tabular}

^ Includes 12 samples collected with TTN group.

Table 5 Sarnat score and 24-48 hour NAG concentrations

\begin{tabular}{lllll}
\hline & Control & Sarnat 1 & Sarnat 2 & Sarnat 3 \\
\hline Number & 55 & 12 & 15 & 7 \\
Mean & 1.89 & 9.28 & 20.71 & 68.54 \\
SD & 1.21 & 9.08 & 14.30 & 144.56 \\
Median & 1.60 & 4.50 & 20.40 & 4.50 \\
$95 \%$ CI & $1.56-2.22$ & $3.52-15.05$ & $12.79-28.63$ & $-65.16-202.24$ \\
\hline
\end{tabular}

$\mu \mathrm{mol} / 1$ at 24 to 48 hours (SD $50.7, \mathrm{n}=33$, range 41-252) and $63 \mu \mathrm{mol} /$ litre (SD 22.3, $\mathrm{n}=21$, range 45-121) at 4-6 days of life.

\section{Discussion}

Traditionally, assessment of perinatal asphyxia has relied on a combination of clinical observations, such as Apgar score, and measurement of systemic indices of tissue ischaemia, such as serum creatinine. There are weaknesses in such methods.

Clinical scoring systems generally reflect neurological state (encephalopathy) which may be influenced by factors other than perinatal asphyxia, such as metabolic and chromosomal disorders. Objective measurements, such as serum creatinine, are insufficiently sensitive to be generally useful. The first form of assessment lacks specificity in the context of perinatal asphyxia; the latter lacks sensitivity.

Perlman ${ }^{8}$ compared several systemic manifestations of asphyxia, including oliguria, serum creatinine, seizures, and cranial ultrasound abnormalities and found that urinary $\beta_{2} \mathrm{M}$ was the most sensitive index of systemic organ injury caused by perinatal asphyxia. Fernandez et al found that urinary $\beta_{2} M$ was more sensitive and specific than "usual tests of renal function" after perinatal asphyxia. Kojima $^{2}$ showed raised NAG in a group of asphyxiated infants over the first week of life, a finding which agrees with our results. ${ }^{10}$

Our data suggest that both urinary NAG and $\beta_{2} M$ are significantly increased in asphyxiated compared with normal term neonates over the first 24 to 48 hours of life. At 4 to 6 weeks there is no statistical difference between the groups, suggesting resolution of hypoxic injury to the renal tubules over this time.

Serum creatinine concentrations have been considered to be indices of perinatal asphyxia. But interpretation is complicated by the fact that for at least the first 48 hours of life, serum creatinine, to a significant extent, reflects maternal concentrations. ${ }^{11}$ Raised values were found in infants with perinatal asphyxia in our study at 24 to 48 hours of life, although the range was wide. The mean value at 4 to 6 days falls within the newborn reference interval. Renal tubular dysfunction is known to occur more commonly than glomerular dysfunction in perinatal asphyxia, ${ }^{5}$ further suggesting a relative insensitivity of serum creatinine compared with indices of tubular function such as urinary $\beta_{2} \mathrm{M}$ and NAG.

Haematuria has been associated with perinatal asphyxia. Tack ${ }^{12}$ found chemical haematuria in $71 \%$ of 140 sick term and premature infants, most of whom were asphyxiated. However, a significant number of non-asphyxiated babies also had haematuria. In our group of term neonates with perinatal asphyxia, 13/32 had no haematuria, including one severely asphyxiated infant who died on the fourth day of life, and $18 / 32$ who had no more than "small" blood on any occasion. It should also be noted that chemical methods for detecting haematuria are non-specific, picking up myoglobin and haemoglobin. In our babies the presence of haematuria on dipstick testing is relatively insensitive for identifying perinatal asphyxia.

None of the infants studied received tolazoline or indomethacin, both of which may injure the newborn kidney. However, use of gentamicin which may be nephrotoxic introduces a possible confounder, ${ }^{13}$ although blood concentrations were within the therapeutic range in all subjects. Our data show an increase in urinary NAG at 24 to 48 hours in both groups treated with gentamicin (perinatal asphyxia and TTN groups) but a greater increase in the former. The results show a positive correlation between NAG and Sarnat score at both 1 to 2 and at 4 to 6 days of life. All babies received similar treatment with gentamicin but NAG rose in proportion to the severity of neonatal asphyxia, suggesting that the antibiotic effect is not the predominant contributory factor.

An objective and semiquantitative index of perinatal asphyxia would be a helpful addition to current techniques for assessing asphyxiated or encephalopathic neonates. In certain circumstances clinical scoring systems are influenced by factors other than perinatal asphyxia without an ability within the scoring system to identify possible aetiologies. With the development of "rescue therapies" for use in perinatal asphyxia, it may be useful to be able to predict at a relatively early stage which infants are likely to benefit most from these treatments that may carry significant risks.

Both $\beta_{2} M$ and NAG are sensitive indices of renal tubular dysfunction in asphyxiated term neonates. Their values probably reflect systemic organ damage produced by an asphyxial insult. Urinary NAG and $\beta_{2} M$ may be useful markers of asphyxia in addition to clinical indicators such as Apgar and Sarnat scores.

Our data suggest that unlike $\beta_{2} M$, urinary NAG increases proportionate to the degree of encephalopathy/asphyxia, as defined by Sarnat score, an association present at both 24 to 48 hours and 4 to 6 days of life. NAG is also more attractive than $\beta_{2} \mathrm{M}$ due to its lower cost.

Measurement of urinary NAG in the first days of life may assist in the diagnosis and assignment of prognosis to an asphyxiated infant, although further studies are required to test these findings. 
We thank Dr TJ Beattie (Royal Hospital for Sick Children, Yorkhill, Glasgow, Scotland) for his helpful comments.

1 Talosi G, Streitman K, Suranyi A, Horvath I, Pinter S. Renal injury in perinatal hypoxia: changes of renal function and injury in perinatal hypoxia: changes of renal function

ultrasonography. Pediatric Nephrol 1996;10:C156.
2 Kojima T, Kobayashi T, Matsuzaki S, Iwase S, Kobayashi Y. Effects of perinatal asphyxia \& myoglobinuria on development of acute neonatal renal failure. Arch Dis Child 1985;60:908-12.

3 Karlowicz MG, Adelman RD. Nonoliguric and oliguric acute renal failure in asphyxiated term neonates. Pediatric Nephrol 1995;9:718-22.

4 Watanabe K, Kojima T, Fukuda Y, Ohbayashi K, Kobayash T, Iwase S, Kobayashi Y. Reliability of urinary N-acetyl-BD-glucosaminidase as an indicator of renal tubular damage in neonates. Biol Neonate 1987;52:16-21.

5 Roberts DS, Haycock DB, Dalton RN, Turner C, Tomlinson P, Timmler L, Scopes JW. Prediction of acute renal failure after birth asphyxia. Arch Dis Child 1990;65:1021-8

6 Tsukahara H, Yoshimoto M, Saito M, Sakaguchi T, Mitsuyoshi I, Hayashi S, et al. Assessment of tubular function in neonates using urinary Beta-2-microglobulin. Pediatric Nephrol 1990;4:512-14.

7 Sarnat HB, Sarnat MS. Neonatal encephalopathy following fetal distress. Arch Neurol 1975;33:669-705.

8 Perlman JM, Tack ED, Martin T, Shackelford G, Amon E. Acute systemic organ injury in term infants after asphyxia. Am 7 Dis Child 1989;143:617-20.

9 Fernandez F, Barrio V, Guzman J, Huertas MD, Zapatero M, de Miguel MD, Mallol J. Beta-2-microglobulin in the assessment of renal function in full term neonates following perinatal asphyxia. $\mathcal{F}$ Perinat Med 1989;17:453-9.

10 Willis FR, Summers J, Minutillo C, Hewitt I. Urinary indices of renal dysfunction in neonatal asphyxia. European Society for Paediatric Research Annual Meeting, Edinburgh, 1993. Pediatr Res 1994;35:281.

11 Mehta KP. Neonatal renal failure. Indian Pediatrics 1991;28:7-9.

12 Tack ED, Perlman JM, Robson AM. Renal injury in sick newborn infants: a prospective evaluation using microglobulin concentrations. Pediatrics 1988;81:432-9.

13 Mathew TM. Drug induced renal disease. Med f Aust 1992;156:724-8. 